\title{
Performance Analysis of LDPC Coded DMT Systems with Bit-loading Algorithms for Powerline Channel
}

\author{
Kyong-Hoe Kim, Seong-Cheol Kim \\ Institute of New Media and Communications \\ School of Electrical Engineering and Computer Science \\ Seoul National University, Seoul, Korea \\ \{khkim, sckim\}@maxwell.snu.ac.kr
}

\begin{abstract}
This paper deals with two different approaches to overcome unfavorable channel characteristics and to obtain higher performance of PLC systems: Low Density Parity Check (LDPC) coding and bit-loading algorithms for Discrete Multitone (DMT) modulation. We analyze the performance of LDPC coded DMT systems with bit-loading algorithms in terms of bit-error rate and data rate. Simulations are performed with statistically modeled in-home PLC channel and noise. Several bit-loading scenarios and LDPC coding schemes are also considered. The performances of the proposed DMT systems with and without LDPC codes are analyzed for the broadband PLC system design.
\end{abstract}

Keywords-Bit-loading, DMT, LDPC, PLC

\section{INTRODUCTION}

$\mathrm{I}^{\mathrm{N}}$ $\mathrm{N}$ recent years, broadband Power Line Communication (PLC) systems have drawn a huge amount of research interest to develop high performance, yet cost efficient, digital data transmission systems. Nowadays, broadband PLC systems are considered as an inexpensive and effective alternative to Digital Subscriber Line (DSL), cable and wireless technologies. However, power supply networks are not designed for communication purposes and they do not offer favorable channel characteristics. The powerline channel is characterized by a large and frequency-dependent attenuation, multipath caused by reflections at discontinuities of networks, time varying impedance and hostile noise conditions.

To overcome unfavorable channel properties of powerline transmission medium, PLC systems have to adopt efficient modulation techniques such as Orthogonal Frequency Division Multiplexing (OFDM) or DMT. Especially, DMT modulation, which has been selected as standard for transmission over DSL, is considered as a suitable solution owing to their abilities in resolving channel impairments to achieve a high transmission capacity. Unlike OFDM which assigns the same number of bits to each subchannel, DMT systems dynamically assign variable bits to subchannel depending on the signal-to-noise ratio (SNR) on each subchannel, that is, more bits to subchannel with higher

This work was supported in part by University IT Research Center project (ITRC) and UPLC Power IT project.
SNR. Assuming the perfect Channel State Information (CSI) at the transmitter, energies and bits are efficiently distributed on each subchannel, which is determined by a so called bit-loading algorithm to achieve either high throughput or high robustness. Under the total transmission power constraint, the optimal bit and energy allocation can be obtained by the well-known water-filling algorithm [1].

To improve bit error probabilities which are caused by high speed data transmission over unfavorable and noisy powerline channel, Forward Error Correction (FEC) techniques must be combined with DMT systems. Low Density Parity Check (LDPC) code [2] is one of the most promising error correcting codes because of its excellent performance. LDPC code was adopted to several standardizations, such as DVB-S2, IEEE 802.11n, and IEEE 802.16e (WiMax). Especially, the Korean standard on high speed powerline communications adopted LDPC codes as an error-correcting code in 2006 [3].

Some authors recently investigated coded DMT systems with bit-loading algorithm over the powerline channel. The performance of OFDM systems with channel coding algorithms including convolutional codes and Reed-Solomon codes was analyzed with statistically representative PLC channels in [4]. But, the proposed system loaded bits to subcarrier based on the table, which has fixed values of thresholds, not on bit-loading algorithms. Turbo coded DMT system and bit-loading algorithms proposed in [5] showed simply that the introduction of turbo codes improves the BER performance.

In this paper, we dealt with LDPC coded DMT systems with a rate adaptive bit-loading algorithm. The rate adaptive bit-loading algorithm [6] maximizes the total transmitting data rate with the given probability of error and the total transmission energy. On the other hand, LDPC codes, which have a powerful error correcting performance, reduce the probability of error. Analyzing how these two different approaches improve jointly the performance, statistically modeled powerline channels are considered to obtain numerous simulation results in terms of bit-error rate and data rate.

The rest of the paper is structured as follows. DMT and the bit-loading algorithm are described in Section II. Powerline 
channel and noise models used in this work are presented in Section III. In Section IV, we introduce the proposed LDPC coded DMT system model. The performance evaluation based on simulations for various scenarios is presented in Section V. Finally, the paper will be summarized in Section VI.

\section{DESCRIPTION OF THE AlgORITHMS}

\section{A. DMT}

DMT is an application of OFDM for a wired channel with efficient bit-loading techniques. The basic idea of DMT is to divide the available bandwidth into a fixed number of $N$ parallel, independent, and equally spaced subchannels. Because the behavior of the wired channel does not change significantly with time, the channel is considered as a stationary during one symbol duration, which makes it possible to apply bit-loading techniques. To assign different numbers of bits to different subchannels, multi-level modulation schemes such as $M$-QAM are used for each subchannel. A detailed description of DMT is given in [7].

The SNR for each subchannel is given by

$$
S N R_{i}=\frac{\varepsilon_{i}\left|H_{i}\right|^{2}}{\sigma_{i}^{2}},
$$

where $\varepsilon_{i}$ and $\left|H_{i}\right|^{2}$ represent the signal energy and the power spectral density of the $i$ th subchannel, respectively, and $\sigma_{i}^{2}$ is the noise variance on the $i$ th subchannel.

The bits per each subchannel is given by

$$
b_{i}=\frac{1}{2} \log _{2}\left(1+\frac{S N R_{i}}{\Gamma}\right),
$$

where the parameter $\Gamma$ is the SNR gap, which indicates how far the system is from the maximum achievable capacity. In other words, it measures the SNR loss from the theoretical maximum channel capacity. Thus, the SNR gap is a performance measure that accounts for both the coding gain and error margin in the performance analysis. The gap depends on a given target probability of symbol error $P_{e}$ and the coding scheme employed. The gap can be reduced by using improved coding scheme when $P_{e}$ is fixed, or accepting a higher $P_{e}$.

The total number of bits transmitted in one DMT symbol, $b$ is

$$
b=\sum_{i=1}^{N} b_{i}=\frac{1}{2} \sum_{i=1}^{N} \log _{2}\left(1+\frac{S N R_{i}}{\Gamma}\right) .
$$

And the achievable bit rate $B$ can be calculated by dividing the total number of bits with the symbol period $T$,

$$
B=\frac{1}{T} b=\frac{1}{T} \cdot \frac{1}{2} \sum_{i=1}^{N} \log _{2}\left(1+\frac{S N R_{i}}{\Gamma}\right) .
$$

\section{B. Rate Adaptive Bit Loading Algorithm}

The main goal of the bit-loading algorithm is to assign bits and energies according to the capacity of subchannels. As well known, the water-filling algorithm generates the optimal energy distribution and achieves theoretical capacity. However, it has been difficult to implement in practice. There are two types of algorithms to suboptimize this problem: Rate-Adaptive (RA) bit-loading algorithm and Margin Adaptive (MA) bit-loading algorithm. The RA bit-loading algorithm [6] maximizes the data rate with a given energy constraint and the MA bit-loading algorithm [8] minimizes the energy with a given data rate.

Since recent broadband communication systems need faster bit rate and probable bit errors can be suppressed using the powerful channel coding scheme and the focus of this paper is the analysis of the performance over broadband powerline channels, we consider a coded DMT system with the RA bit-loading using the Leke-Cioffi algorithm [6] in this work.

As stated above, The RA bit-loading algorithm maximizes the number of bits per dimension subject to a fixed energy constraint:

$$
\max _{E_{i}} b=\frac{1}{2} \sum_{i=1}^{N} \log _{2}\left(1+\frac{\varepsilon_{i} \cdot g_{i}}{\Gamma}\right),
$$

subject to a given total energy budget $\varepsilon=\sum_{i=1}^{N} \varepsilon_{i}$,

where $g_{i}=\left|H_{i}\right|^{2} / \sigma_{i}^{2}$, which represents the SNR with a unit energy of a subchannel.

It is proven in [6] that the design objective is achieved when,

$$
\varepsilon_{i}+\frac{\Gamma}{g_{i}}=\text { constant. }
$$

The term "water-filling" describes this solution. The curve of inverse SNR $\Gamma / g_{i}$ is being a bowl into which the water (energy) is poured, filling the curve until there is no more energy to use.

In the Leke-Cioffi algorithm, subchannels with SNR below a certain threshold are completely "turned off" or nulled. The first step of this algorithm is to determine which channels should be turned off. Subchannel $m$ will be turn off if

$$
\Gamma \cdot \frac{\sigma_{m}^{2}}{\left|H_{m}\right|^{2}}>\frac{1}{N_{\text {on }}} \cdot\left(\varepsilon+\Gamma \cdot \sum_{n=1}^{N_{\text {on }}} \frac{\sigma_{n}^{2}}{\left|H_{n}\right|^{2}}\right),
$$

where $N_{\text {on }}$ is the number of subchannels turned on.

The water-filling distribution of energies can be obtained by solving the following two equations, iteratively:

1. The constant level $K$ is given by

$$
K=\frac{1}{N} \cdot\left(\varepsilon+\Gamma \cdot \sum_{n=1}^{N} \frac{1}{g_{n}}\right) .
$$

2. The energy in each subchannel turned on 


$$
\varepsilon_{n}=K-\frac{\Gamma}{g_{n}}
$$

for $n=1, \ldots, N$.

If one or more of $\varepsilon_{n}<0$, then the most negative is eliminated and (8) and (9) are solved again with $N \rightarrow N-1$ and the corresponding $g_{n}$ term eliminated.

Finally, the number of bits per subchannel is obtained from

$$
b_{n}=\frac{1}{2} \log _{2}\left(1+\frac{\varepsilon_{n} \cdot g_{n}}{\Gamma \cdot \gamma_{m}}\right),
$$

where $\gamma_{m}$ is the target margin. The margin is the amount by which the SNR on the channel may be lowered before performance degrades to a probability of error greater than the target $P_{e}$ used in defining the gap. The bits $b_{n}$ have to be rounded to the integer bits $B_{n}$ due to a finite granularity and the energy $\varepsilon_{n}$ have to be rescaled to $E_{n}$ accordingly.

\section{Powerline Channel And Noise Model}

There are frequency dependent attenuations and deep narrowband notches in the powerline channel transfer function. These are caused by multiple reflections at impedance discontinuities. Thus, we can simplify the channel with a multipath model. In this case, the impulse response function of the channel can be modeled as a sum of weighted Dirac-impulses,

$$
h(\tau ; t)=\sum_{p=0}^{N_{p}-1} \alpha_{p}(t) \cdot \delta\left(\tau-\tau_{p}(t)\right),
$$

where $\alpha_{p}$ is the channel attenuation coefficient of $p$ th path, $\tau_{p}$ is the delay of $p$ th path, and $N_{p}$ is the number of path. To get the values of these channel parameters, a simple statistical method, which has been presented in [9], is used for this work. Using as the in-home powerline reference channels, default values for four different quality classes listed in Table I. On the restriction of these default values, impulse responses are generated in a random manner. A detailed description is given in [9].

Noises in the powerline can be classified mainly into two categories, namely the background noise and the impulsive noise. The background noise has a power spectral density which decreases with frequency, and is usually stationary over relatively longer periods, of seconds, minutes and sometimes even of some hours. The amplitude of the background noise is relatively much lower than the amplitude of the impulsive noise, thus, the background noise is omitted in our simulation.

Because of its higher amplitudes, impulsive noise is considered the main cause of burst error occurrence in data transmission over the high frequencies of the powerline medium. The Middleton's Class A noise model [10] is considered as a statistical model of impulsive noise. This model is composed of

\begin{tabular}{|c|c|c|c|c|c|}
\hline Model & $\begin{array}{l}\text { Number } \\
\text { of paths }\end{array}$ & $\begin{array}{l}\text { Impulse } \\
\text { response } \\
\text { duration }\end{array}$ & $\begin{array}{l}\text { Initial } \\
\text { delay }\end{array}$ & $\begin{array}{l}\text { Maximum } \\
\text { amplitude }\end{array}$ & $\begin{array}{l}\text { Minimum } \\
\text { amplitude }\end{array}$ \\
\hline "excellent" & 5 & $0.5 \mu \mathrm{s}$ & $0.2 \mu \mathrm{s}$ & 0.05 & 0.005 \\
\hline “good" & 10 & $1.0 \mu \mathrm{s}$ & $0.2 \mu \mathrm{s}$ & 0.01 & 0.002 \\
\hline "medium" & 15 & $1.5 \mu \mathrm{s}$ & $0.5 \mu \mathrm{s}$ & 0.003 & 0.003 \\
\hline "bad" & 20 & $2.0 \mu \mathrm{s}$ & $0.5 \mu \mathrm{s}$ & 0.0005 & 0.0001 \\
\hline
\end{tabular}
the sum of background Gaussian noise and impulsive noise.
TABLE I

According to the Middleton's Class A noise model, the normalized complex probability density function (PDF) of the model is given by

$$
\begin{gathered}
p_{z}(z)=\sum_{m=0}^{\infty} \frac{e^{-A} A^{m}}{m !} \cdot \frac{1}{2 \pi \sigma^{2}} \exp \left(-\frac{|z|^{2}}{2 \sigma_{m}^{2}}\right), \\
\text { with } \sigma_{m}^{2}=\sigma^{2} \cdot \frac{m / A+\Gamma_{\mathrm{GIR}}}{1+\Gamma_{\mathrm{GIR}}}
\end{gathered}
$$

where $z=x+j y$ is the complex envelop, $x$ is the in-phase component, $y$ is the quadrature component of the noise, and $A$ is the impulsive index, and $\Gamma_{\mathrm{GIR}}\left(=\sigma_{g}^{2} / \sigma_{i}^{2}\right)$ is the Gaussian-to-Impulsive power Ratio (GIR) with the Gaussian noise power $\sigma_{g}^{2}$ and the impulsive noise power $\sigma_{i}^{2}$, and $\sigma^{2}=\sigma_{g}^{2}+\sigma_{i}^{2}$ is the total noise power.

\section{LDPC CODED DMT SYSTEM}

A block diagram of the LDPC coded DMT system with the bit-loading algorithm in this simulation is shown in Fig. 1. In this section, simulation environments presented in Fig. 1 is described block by block. The following conditions have been assumed in the proposed system:

- Ideal synchronization at the receiver;

- The channel state information is totally known both at the transmitter and at the receiver;

- Bandwidth ranging from 1 to $41 \mathrm{MHz}$;

- Number of subchannels $N$ equal to 1024 .

\section{A. LDPC Encoding}

A LDPC codes is a linear block code with a parity-check matrix that is sparse, that is, it has a small number of nonzero entries. If the number $w_{c}$ of 1 's in each column is constant and the number $w_{r}$ of 1 's in each row is also constant for every row, the code is called a $\left(w_{c}, w_{r}\right)$ regular LDPC code. On the other hand, if the parity-check matrix has low density, but the numbers of 1's in each row or column aren't constant, the code is called an irregular LDPC code. It has been found that irregular LDPC codes show much better performance close to the capacity than regular ones [11]. And then, in [12], Richardson and Urbanke developed a density evolution algorithm to analyze and design the irregular LDPC codes. To compare the performance improvement with different coding schemes, we 


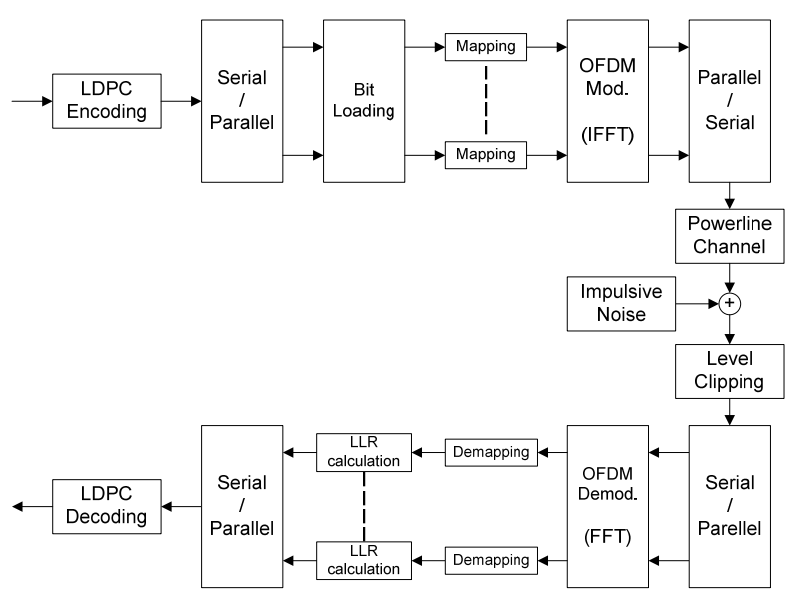

Fig. 1. Block diagram of LDPC coded DMT system

TABLE II

DEGREE DISTRIBUTION OF IRREGULAR LDPC CODE

\begin{tabular}{|c|c|c|c|c|c|}
\hline Degree & 2 & 3 & 4 & 6 & 7 \\
\hline Probability & 0.45782 & 0.32377 & 0.02142 & 0.0592 & 0.0389 \\
\hline \hline Degree & 8 & 9 & 19 & 20 & \\
\hline Probability & 0.02481 & 0.00884 & 0.01767 & 0.04746 & \\
\hline
\end{tabular}

present two LDPC codes in this simulation. The first one is the $(3,6)$ regular code whose code length is 1024 and code rate is $1 / 2$. The second one is the irregular code whose code rate is also $1 / 2$, but code length is 4096 , four times longer than the first one. Thus, the second code must be more powerful error correcting code compared to the first one. The degree distribution of the irregular code is chosen by the density evolution algorithm [12], and is shown in Table II.

\section{B. Bit-loading and Mapping}

After the LDPC encoding of information source bits, bits and energies are dynamically allocated to each subchannel by using the RA bit-loading algorithm presented in Section II. Depending on the SNR of the subchannel, 0 to 10 bits are mapped to each subchannel. For the modulation of the subchannel, BPSK (for 1 bit per subchannel) and gray coded rectangular $M$-QAM $\left(M=4,8,16,64,256\right.$, and 1024 , which assigns $\beta\left(\triangleq \log _{2} M\right)$ bits per subchannel, respectively) is used. The process after the mapping is the same as a typical OFDM transceiver, and is illustrated in Fig. 1.

\section{Powerline Channel and Impulsive Noise}

In our simulation, four in-home reference channels, which have different qualities, in Section III, are considered. Reference channels are randomly generated by using the default values in Table I.

The impulsive noise is also randomly generated with the impulsive index $A=0.1$ and the GIR $\Gamma_{\mathrm{GIR}}=0.1$. The ratio of energy per bit to the noise spectral density for the $i$ th subchannel is calculated by

$$
\left\{\frac{E_{b}}{N_{0}}\right\}_{i}=S N R_{i} \cdot \frac{1}{B_{i}} \cdot \frac{1}{R_{C}},
$$

where $S N R_{i}$ is the SNR from (1), $B_{i}$ is the allocated bits which is rounded to an integer from (10), and, thus, is one of the value $\{1, \beta\}$, and $R_{C}$ is the code rate of the LDPC code. Then, the ratio of energy per bit to the noise spectral density for the entire OFDM symbol is given by

$$
\left\{\frac{E_{b}}{N_{0}}\right\}_{\text {OFDM }}=\frac{1}{N} \sum_{i=1}^{N}\left\{\frac{E_{b}}{N_{0}}\right\}_{i} .
$$

\section{Impulsive Noise Mitigation}

Multi-carrier systems such as DMT are more resistant to the effects of the impulsive noise compared to single carrier systems. However, the impulsive noise can still cause significant problems in multi-carrier systems [13]. Several authors have used techniques for mitigating the effect of impulsive noise by clipping impulsive peaks. The background of the clipping technique is described well in [14]. Especially, authors in [15] used this technique for the LDPC coded OFDM system. The clipping technique applied on the proposed system is a simple non-linear function in the received time domain. Clipping is executed both at the real part and at the imaginary part of the received DMT signal samples as follows:

$$
r_{l, \text { clipped }}=\left\{\begin{array}{cl}
A_{\text {clip }}, & r_{l} \geq A_{\text {clip }} \\
r_{l}, & \left|r_{l}\right| \leq A_{\text {clip }}, \\
-A_{\text {clip }}, & r_{l} \leq-A_{\text {clip }}
\end{array}\right.
$$

where $r_{l}$ is the real or imaginary part of the received DMT signal samples at time $l, A_{\text {clip }}$ is the clipping level normalized with the transmitted DMT signal amplitude. The value of $A_{\text {clip }}$ is fixed to 1.0 in our simulations.

\section{E. LDPC Decoding}

After the FFT demodulation at the receiver, initial Log-Likelihood Ratio (LLR) must be calculated because the LDPC decoding algorithm requires the previous computation of the bit soft information, that is, LLR as a priori for every bit of a codeword. The LLR is defined as the ratio of two conditional a posteriori probabilities,

$$
\operatorname{LLR}\left(d_{k, j}\right)=\ln \frac{\operatorname{Pr}\left(d_{k, j}=1 \mid r(k), H(k), \sigma^{2}\right)}{\operatorname{Pr}\left(d_{k, j}=0 \mid r(k), H(k), \sigma^{2}\right)},
$$

where $d_{k, j}$ represents $j$ th bit in the $k$ th subchannel which assigns $M$-QAM symbol $X_{k}=\Lambda\left(b_{k, 1}, b_{k, 2}, \ldots, b_{k, \beta}\right) \in S$, where $\beta=\log _{2} M, \Lambda$ is a function which maps $\beta$ channel encoder output bits $c_{i}(i=1,2, \ldots, \beta)$ into one $M$-QAM symbol $S \in\left\{s_{1}, s_{2}, \ldots, s_{M}\right\}$, and $r(k)$ is the FFT output signal for the $k$ th 
subchannel. Since the clipped signal $r_{l, \text { clipped }}$ in the time domain is approximately Gaussian, the FFT output $r(k)$ of $r_{l, \text { clipped }}$ in the frequency domain is also approximately Gaussian. Thus, the conventional minimum distance metric can be applicable for calculating LLRs. If it is assumed that the transmitted $M$-QAM symbols are equally probable, and given $H(k)$ and $\sigma^{2}$, the LLR can be calculated as

$$
\begin{aligned}
\operatorname{LLR}\left(d_{k, j}\right)= & \ln \sum_{s_{i} \in\left\{s: c_{j}=1\right\}} \exp \left\{-\left|r(k)-H(k) \cdot s_{i}\right|^{2} / 2 \sigma^{2}\right\} \\
& -\ln \sum_{s_{i} \in\left\{s: c_{j}=0\right\}} \exp \left\{-\left|r(k)-H(k) \cdot s_{i}\right|^{2} / 2 \sigma^{2}\right\}
\end{aligned}
$$

Suboptimal simplified LLR with the log-sum approximation $\ln \sum_{j} \exp \left\{z_{j}\right\} \approx \max _{j} \ln \left(\exp \left\{z_{j}\right\}\right)=\max _{j} z_{j}$ can be given as

$$
\begin{aligned}
\operatorname{LLR}\left(d_{k, j}\right) \approx & \frac{1}{2 \sigma^{2}}\left(\max _{s_{i} \in\left\{s: c_{j}=1\right\}}\left\{-\left|r(k)-s_{i} \cdot H(k)\right|^{2}\right\}\right. \\
& \left.-\max _{s_{i} \in\left\{s: c_{j}=0\right\}}\left\{-\left|r(k)-s_{i} \cdot H(k)\right|^{2}\right\}\right)
\end{aligned}
$$

Given LLR in (19), the LDPC decoding is accomplished by using the message-passing algorithm [16]. The maximum number of decoding iteration was set to 20 in our simulation.

\section{Simulation Results}

\section{A. Performances of Uncoded DMT System}

Before the LDPC coded system is considered, the performance of the uncoded DMT System is described first in terms of Bit-Error Rate (BER) and data rate. The RA bit-loading algorithm is applied and the SNR gap $\Gamma$ is set to $9.0 \mathrm{~dB}$ in this simulation.

Data rate and BER for four in-home powerline reference channels are illustrated in Fig. 2 and Fig. 3, respectively. The results are slightly different according to the channel model. Fig. 2 shows that data rate is increased exponentially with respect to the $E_{b} / N_{0}$. Note that a logarithmic axis is used for the data rate. As the SNR per each subchannel is increased, the RA bit-loading algorithm assigns more bits on the subchannel. Since there is a limit for the maximum bits per subchannel, 10 bits in our system, according as the $E_{b} / N_{0}$ is increased, the number of saturations occurred in subchannels, which have more favorable SNR conditions than others, is increased. Thus, the BER is decreased more rapidly when most of subchannels are saturated, as shown in Fig 3. However, to obtain a moderate level of BER, a very high value of $E_{b} / N_{0}$, which is unachievable at a realistic powerline channel, is required.

\section{B. Performances of LDPC Coded DMT System}

As mentioned in section IV, two LDPC codes are chosen in this paper; one is a $(3,6)$ regular code with length 1024

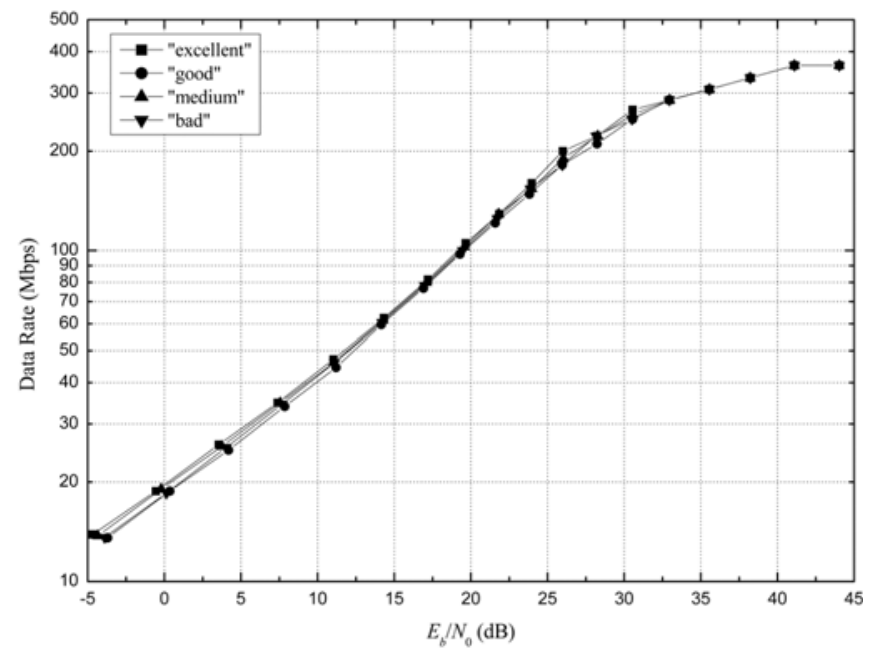

Fig.2. Data rate of uncoded DMT system

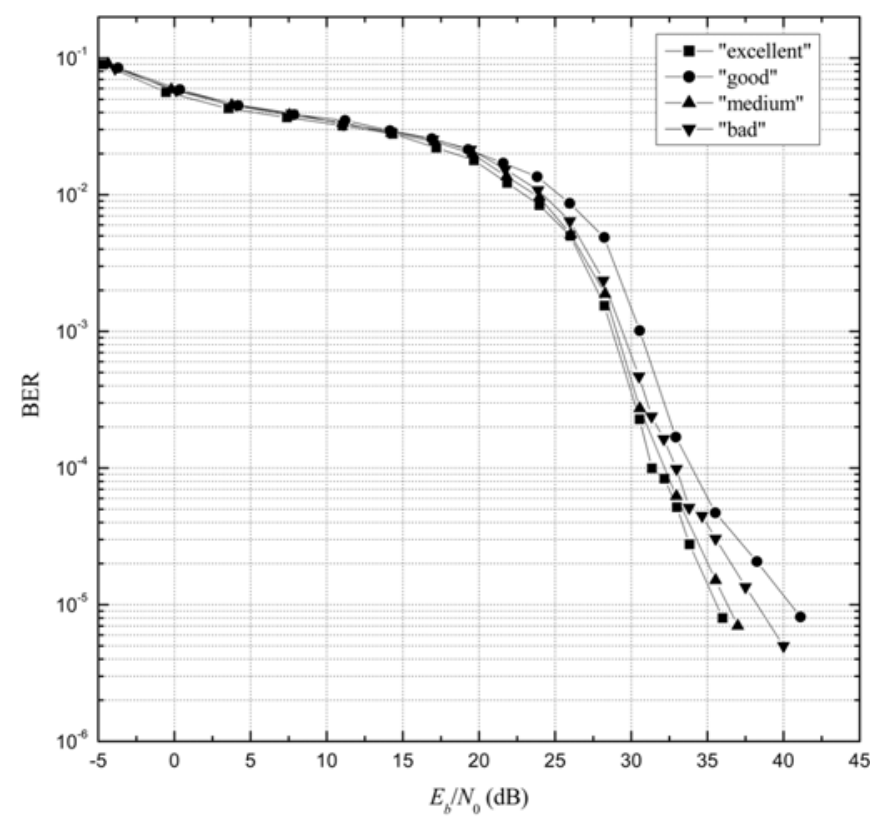

Fig. 3. BER of uncoded DMT system

(referred as Reg.), and another is an irregular code with length 4096 (referred as Irr.). The RA bit-loading algorithm with different values of the SNR gap $\Gamma$ is simulated. The robustness of the RA bit loading algorithm is determined with respect to the SNR gap which is used like a target margin in this simulation. Namely, smaller gap indicates more bits per each subchannel. Thus, more powerful codes are capable of having smaller gap.

Data rate and BER for the in-home powerline reference channel "bad" are represented in Fig. 4 and Fig. 5, respectively. In Fig. 4, the data rate is increased at a given level of $E_{b} / N_{0}$ as the SNR gap is decreased. It is clear that the data rate depends only on the SNR gap and the $E_{b} / N_{0}$, not on the coding scheme. Fig. 5 shows that the BER falls rapidly at a significantly lower level of $E_{b} / N_{0}$ than one of the uncoded system. This effect is 
due to the powerful coding gain of the LDPC code. While the probability of error in the uncoded system is not maintained on the desired value by the RA bit-loading algorithm, the LDPC coded system rapidly drops the probability of error. Obviously, the irregular code with a longer length outperforms the regular code with a shorter length at higher $E_{b} / N_{0}$, as shown in Fig 5.

As the SNR gap is increased at a given level of $E_{b} / N_{0}$, the probability of error is decreased, but the data throughput is also decreased as shown in Fig. 4 and Fig. 5. Therefore, there is a design trade-off between the probability of error and the data throughput with respect to the SNR gap. If the desired value of the error probability is determined in the system, one can find the SNR gap which maximizes the data throughput at the given level of the error probability.

\section{CONCLUSIONS}

In this paper, we simulated performances of a DMT system with the rate adaptive bit-loading algorithm at statistically modeled powerline channels with impulsive noise. The proposed systems with and without LDPC codes were compared in terms of bit-error rate and data rate. Simulation results showed the performance of the DMT system with the rate adaptive bit-loading algorithm is improved by applying the LDPC codes which have a powerful coding gain. It was confirmed that the SNR gap controls the trade-off between the data throughput and the probability of error.

\section{REFERENCES}

[1] R.G. Gallagher, Information Theory and Reliable Communication, Wiley, 1968.

[2] R. G. Gallagher, "Low-density parity-check codes," IRE Trans. Inform. Theory, vol. IT-8, Jan. 1962.

[3] Korean Standard Association, KS X 4600-1: 2006, Information technology-Telecommunications and information exchange between systems Power Line Communications(PLC) - High speed PLC Medium Access Control(MAC) and Physical Layer(PHY) - Part 1 : General requirement, May 2006.

[4] M. Babic, T. Kistner, J. Bausch, and K. Dostert, "Performance Analysis of Coded OFDM Systems at Statistically Representative PLC Channels," in Proc. of the IEEE International Symposium on Power Line Communications and its Applications (ISPLC 2006), Orlando, Florida, USA, Mar. 2006.

[5] S. Morosi, E. Del Re, Romano Fantacci, and D. Forasacchi, "Turbo-coding and Bit-loading Algorithms for a HomePlug-like DMT PLC System," in Proc. of the IEEE International Symposium on Power Line Communications and its Applications (ISPLC 2006), Orlando, Florida, USA, Mar. 2006.

[6] A. Leke and J. M. Cioffi, "A maximum rate loading algorithm for discrete multitone modulation systems," in Proc. of IEEE Global Telecommunications Conference (GLOBECOM '97), vol. 3, Phoenix, Arizona, USA, Nov. 1997.

[7] J. M. Cioffi, "A Multicarrier Primer," ANSI T1E1.4 Committee Contribution, Nov. 1991.

[8] P. S. Chow, J. M. Cioffi, and J. A. C. Bingham, "A practical discrete multitone transceiver loading algorithm for data transmission over spectrally shaped channels," IEEE Trans. on Comm., vol. 43, 1995.

[9] M. Babic, M. Hagenau, K. Dostert, and J. Bausch, OPERA deliverable D4: Theoretical postulation of PLC channel model, Mar. 2005.

[10] D. Middleton, "Statistical-physical model of electromagnetic interference," IEEE Trans. on Electromagn. Compat., vol. EMC-19, Aug. 1977.
[11] M. G. Luby, M. Mitzenmacher, M. A. Shokrollahi, and D. A. Spielman, "Improved low-density parity-check codes using irregular graphs," IEEE Trans. on Inform. Theory, vol. IT-47, Feb. 2001.

[12] T. J. Richardson, M. A. Shokrollahi, and R. L. Urbanke, "Design of capacity-approaching irregular low-density parity-check codes," IEEE Trans. on Inform. Theory, vol. IT-47, Feb. 2001.

[13] M. Ghosh, "Analysis of the effect of impulse noise on multicarrier and single carrier QAM systems," IEEE Trans. on Comm., vol. 44, Feb. 1996.

[14] H. A. Suraweera, C. Chai, J. Shentu and J. Armstrong, "Analysis of impulse noise mitigation techniques for digital television systems," in Proc. of 8th International OFDM Workshop (InOWo'03), Hamburg, Germany, Sep. 2003.

[15] H. M. Oh, Y. J. Park, S. Choi, J. J. Lee, and K. C. Whang, "Mitigation of Performance Degradation by Impulsive Noise in LDPC Coded OFDM System," in Proc. of the IEEE International Symposium on Power Line Communications and its Applications (ISPLC 2006), Orlando, Florida, USA, Mar. 2006.

[16] D.J.C. MacKay, "Good error-correcting codes based on very sparse matrices," IEEE Trans. on Inform. Theory, vol. 45, No. 2, Mar. 1999.

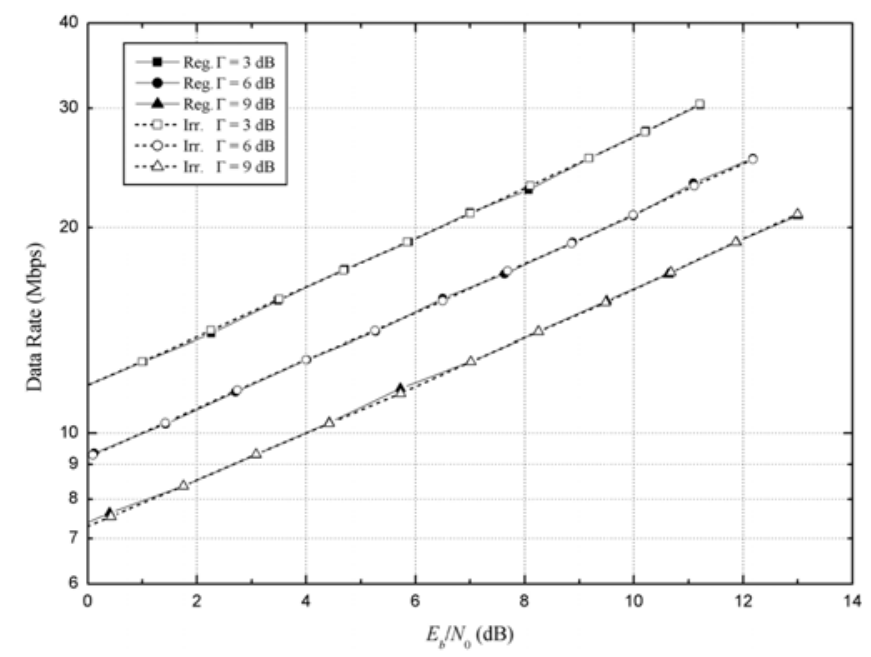

Fig. 4. Data rate of LDPC coded DMT system

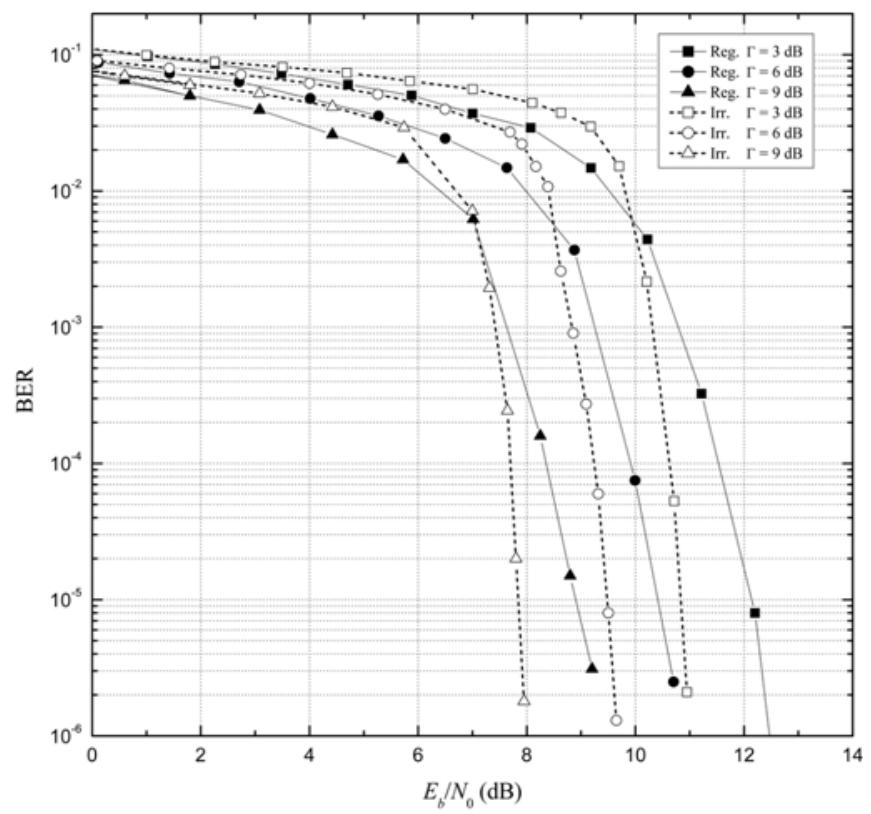

Fig. 5. BER of LDPC coded DMT system 